\title{
Implementation of packet scheduling algorithms in LTE-Sim
}

\author{
Abdallh F. Abdallh, Naif Alharbi, Kumbesan Sandrasegara \\ Faculty of Engineering and Information Technology \\ University of Technology,Sydney \\ Abdallh.F.Abdallh@student.uts.edu.au \\ naif_d@hotmail.com \\ Kumbesan.Sandrasegaran@uts.edu.au
}

\begin{abstract}
In this research paper we have implemented downlink packet scheduling algorithms in LTE-Sim simulation for LTE cellular networks. The implementation includes adding two packet scheduling algorithms (Round Robin and MaxRate). The performance of these algorithms is tested for various types of real time service (video) and non real time service(data) for fixed and mobile users at various speeds 3 , and $120 \mathrm{Km} / \mathrm{h}$ in LTE-Sim simulator. The simulation was setup with varying number of users 120 in fixed boundedregions of $1 \mathrm{~km}$ radius.
\end{abstract}

The goal of this project is to study the simulation of Radio Resource Management (RRM) in 4G networks using LTE-Sim. In particular, it focuses on the comprehensive performance testing of packet scheduling algorithms (such as Max Rate, Round Robin, PF, MLWDF, EXP). The paper shows the results of implementing of these algorithms and testing their performance.

\section{Keywords}

LTE-Sim; Packet scheduling algorithms; Round Robin; MaxRate

\section{Council for Innovative Research}

\author{
Peer Review Research Publishing System
}

\section{Journal: INTERNATIONAL JOURNAL OF COMPUTERS \& TECHNOLOGY}

Vol.14, No.8

www.ijctonline.com, editorijctonline@gmail.com 


\section{INTRODUCTION}

With increasing demand on high data rate and broad mobility, Long Term Evolution (LTE) is introduced by the third generation partnership project (3GPP)to meet that demand[1].It promises to provide $100 \mathrm{Mbps}$ at the downlink through using orthogonal frequency division multiple access (OFDMA) and 50Mbps at the uplink via using Single Carrier Frequency Division Multiple Access (SCFDMA) [2]. The OFDMA technology splits the existing bandwidth into multiple narrow-band subcarriers and assigns a group of sub-carriers to a user based on its necessity, current system load and system configuration. The architecture of LTE network has changed with compared to UMTS as some elements combined together. As shown in figure 1, it consists of three elements: evolved-NodeB (eNodeB), Mobile Management Entity (MME), and Serving Gateway (S-GW) / Packet Data Network Gateway (P-GW). eNodeBcarry outs all radio resource management functions such as packet scheduling and handover mechanism. MME performs mobility, user equipment (UE) identity, and security parameters. SGW and P-GW are nodes that terminate the interface towards E-UTRAN and Packet Data Network, respectively [3].

\section{EPC (Evolved Packet Core)}

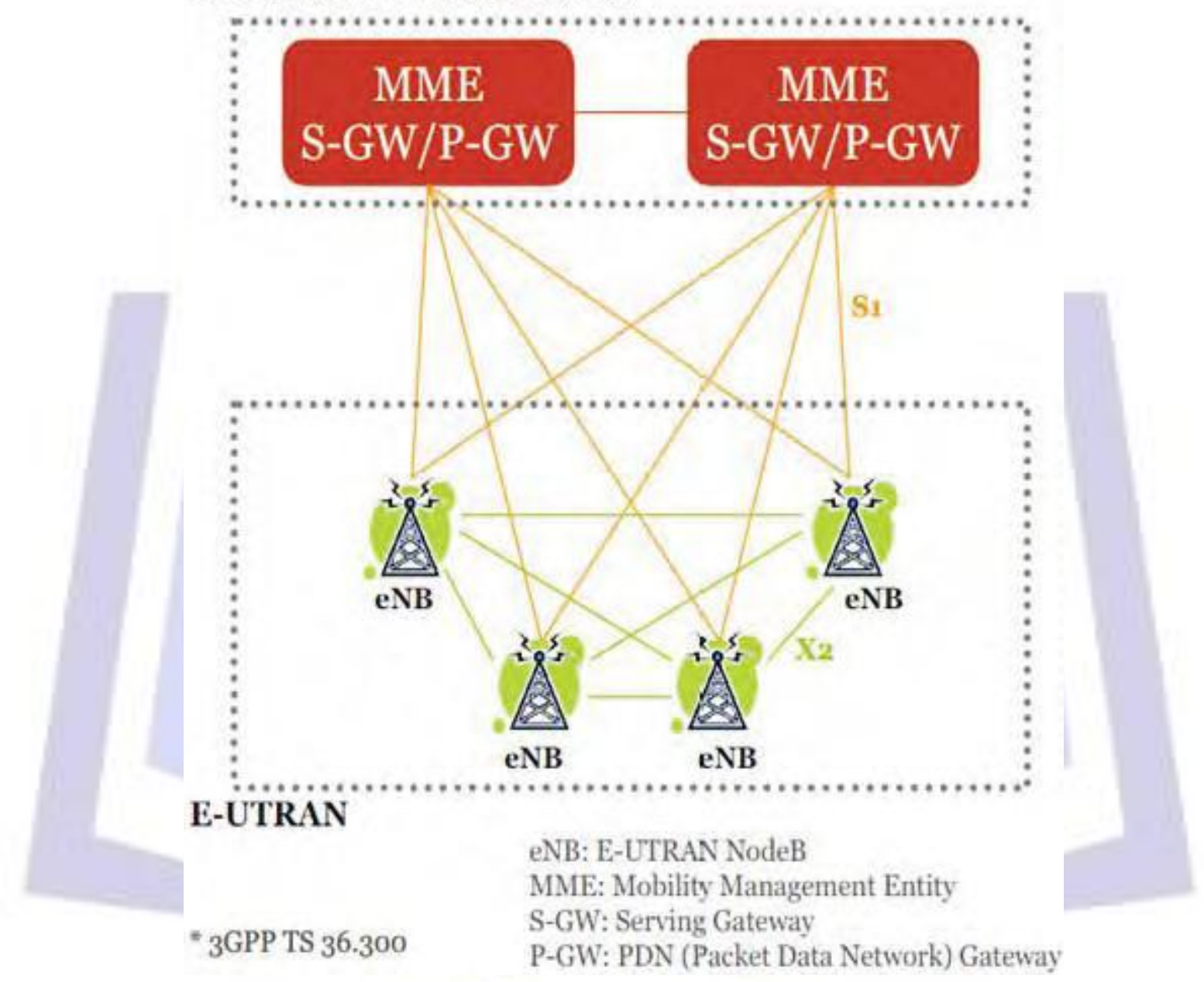

Fig1: LTE system architecture [3]

After implementing the algorithms, this paper will study the performance of packet scheduling and handover algorithms developed for single carrier wireless systems for real time (RT) and non real time (NRT) services. The performance assessment is performed using the LTE-Sim simulationprogram. LTE uses radio resource management which plays a key role in selection of users and transmissions of their packets such that the radio resources are efficiently utilized and the users' qualities of service (QoS) requirements are satisfied[4].

LTE provides user in downlink a resource block $(R B)$ which is defined in frequency and time domain. RB has 12 subcarriers of $180 \mathrm{kHz}$ bandwidth that corresponding to one time slot of $0.5 \mathrm{~ms}$ duration in the time domain. Each time slot has 7 OFDMsymbols. The assigning of this resource is performed every transmit time interval (TTI) that equals to $1 \mathrm{~ms}[5]$.

\section{Packet scheduling algorithms in LTE}

LTE uses packet scheduling algorithms to increase throughput with maintaining fairness. This paper will test and evaluate some of the well known algorithms. 
The maximum rate (Max-Rate)[6] algorithm allocates recourses based on the channel quality indicator (CQI) .The users with highest CQI report will be given resources. This will guaranteed the user will transmit and received data with a good channel condition. However, users with low CQI report will be deprived until their CQI report become high. This will provide low fairness as a result of not all the users will be given resources only the users with highest CQI.Max-Rate depends on the metric value $(\mathrm{K})$ to select user and allocate resources which is calculated based on the following equation:-

$$
\mathrm{K}=\arg \max r(\mathrm{t})
$$

where $r(t)$ : the achievable data rate of user.

To provide some fairness between users, a Round Robin (RR) (1) algorithm is used to solve this issue with Max-Rate algorithm. RR allocates equal amount of resources and time for each user. As RR does not depend on the CQI report, the throughput will be decreased.

Both fairness and high throughput are required .To support both of them and provide balance, proportional fail (PF) [7] algorithm was developed.PF was designed initially to support NRT service and to be used in code division multiple access with high data rate (CDMA-HDR) system. It uses a metric $\mathrm{k}$ in order to allocate resources to users.

$$
k=\arg \max \frac{r_{i}(t)}{R_{i}(t)}
$$

Where ri(t) is the achievable data rate of useri ,and $\mathrm{Ri}(\mathrm{t})$ is the average data rate of useriover a time window (tc) of an appropriate size. This time window guarantees increasing throughput and providing fairness for users.

To support RT servicesin CDMA-HDR system, the maximum-largest weighted delay first (M-LWDF) [8] algorithmis developed. M-LWDF selects user based on the metric that calculated from the following equation:

$$
k=\arg \max a_{i} W_{i}(t) \frac{r_{i}(t)}{R_{i}(t)}
$$

and:

$$
a_{i}=-\frac{\left(\log \delta_{i}\right)}{\tau_{i}}
$$

where $\mathrm{Wi}(\mathrm{t})$ is the head of line $(\mathrm{HOL})$ packet delay (time difference between the current time and the arrival time of a packet) of user i at time $\mathrm{t},{ }^{\delta_{i}}$ is the delay threshold of user $\mathrm{i}$ and ${ }^{\delta_{i}}$ is the maximum probability for HOL packet delay of user $\mathrm{i}$ to exceed the delay threshold of user $\mathrm{i}$.

M-LWDF combines both PF and HOL packet delay in its properties .It provides a good throughput with low packet loss ratio (PLR).

To support multimedia services in anadaptive modulation and coding and time divisionmultiplexing (AMC/TDM) system,the exponential/proportional fair (EXP/PF) [9] algorithm is designed.EXP/PF uses a metric $\mathrm{k}$ which depends on the type of each user whether it is RT or NRT.

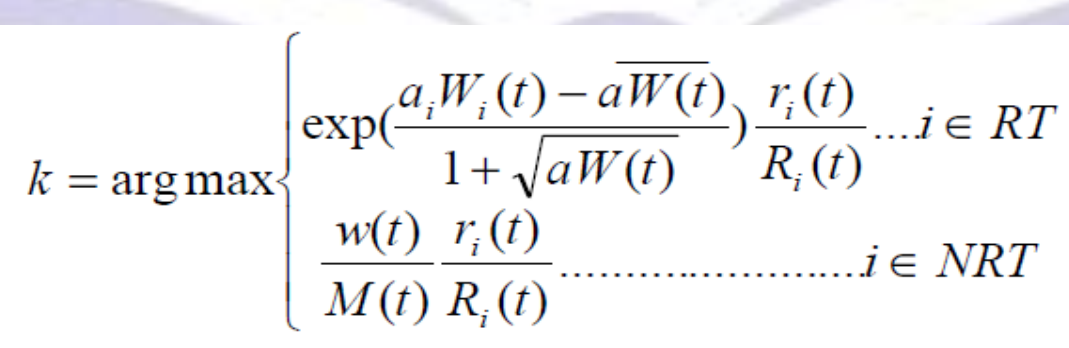

and:

$$
\begin{aligned}
& \overline{a W(t)}=\frac{1}{N_{R T}} \sum_{i \in R T} a_{i} W_{i}(t) \\
& w(t)=\left\{\begin{array}{l}
w(t-1)-\varepsilon \ldots \ldots W_{\max }>\tau_{\text {max }} \\
w(t-1)+\frac{\varepsilon}{k} \ldots . . . W_{\text {max }}<\tau_{\text {max }}
\end{array}\right.
\end{aligned}
$$


Where $M(t)$ is the average number of real time packets waiting at the serving eNodeB buffer at time $t, \varepsilon$ and $k$ are constants, Wmax is the maximum HOL packet delay of all RT service users and $\mathrm{T}$ max is the maximum delay constraint out of RT service users. In the EXP/PF algorithm, real time users receive a higher priority than non real time users when their HOL packet delays are approaching the delay deadline.

\section{Methods}

LTE-Sim is an open source simulation for LTE networks. It contains all the LTE network elements such as, Evolved Universal Terrestrial Radio Access (E-UTRAN) and the Evolved Packet System (EPS).Different scenarios can be implemented for single, mutli-cell environment. Moreover, it supports handover procedures and packet scheduling. Three well-known packet scheduling algorithms are already implemented (such as Proportional Fair, Modified Largest Weighted Delay First, and Exponential Proportional Fair)[4].

In this paper Round Robin (RR) and MaxRate(MR) scheduling algorithms with addition to LTE Standard Hard Handover, Received Signal based TTT Window and Integrator Handover are implemented with along with installed algorithms. For all algorithms, new codes havewritten to enable these new algorithms in LTE-Sim.

\section{Verifying of implementation RR and MR scheduling algorithms}

The following sections prove that the implementations of the packet scheduling algorithms areworking properly.

\subsection{Round Robin}

To make sure the code that has been written satisfy the RR algorithm, a test is carried out to explain how the new algorithm works. The number of resource blocks is set to be 1 for each flow. A simple scenario of five flows is tested with $5 \mathrm{MHs}$ bandwidth (25 RBs). The results are shown in the figure $2 \& 3$ below:

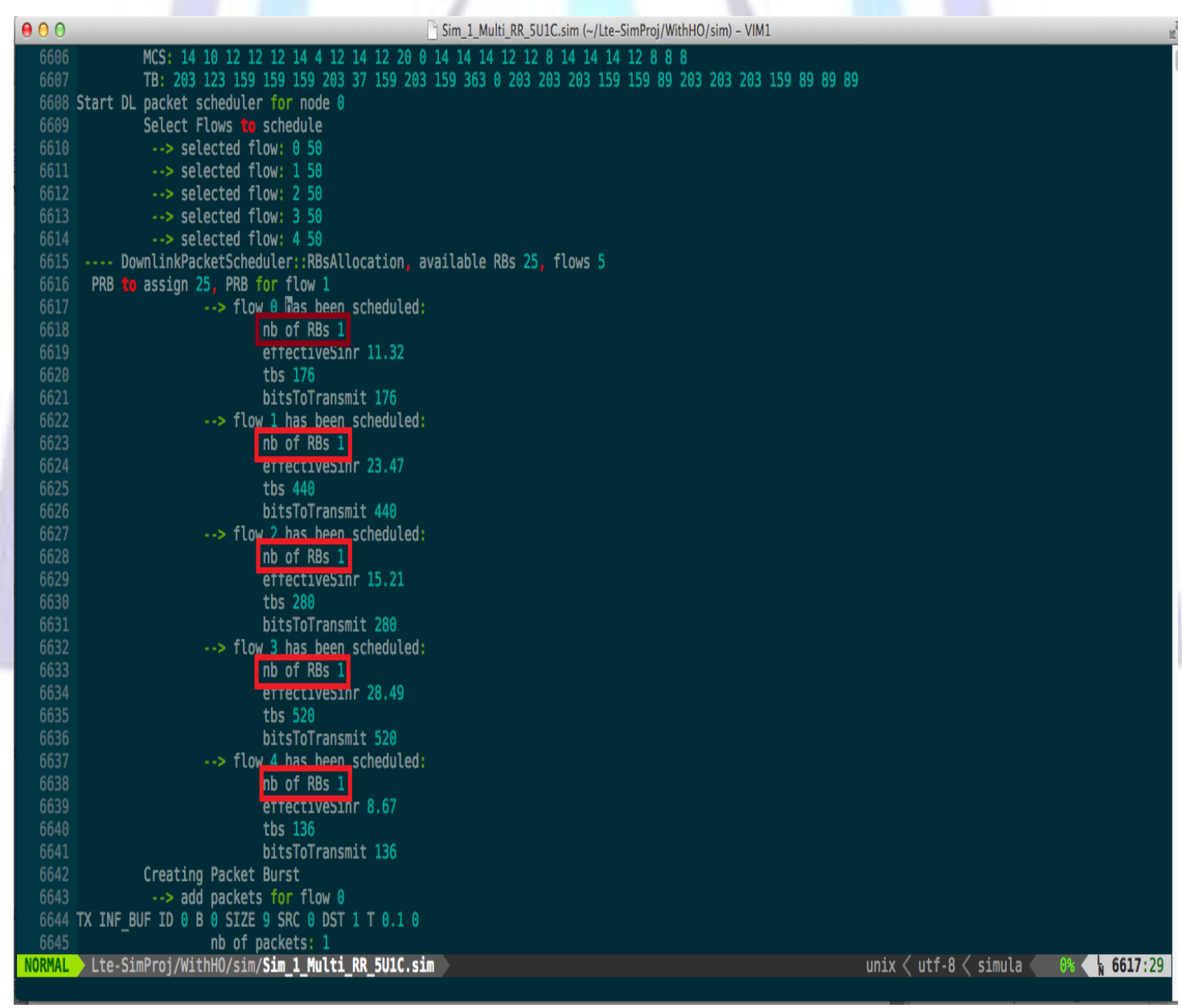

Fig2: RR allocates 1 RB for each flow

Figure 3 shows each flow has assigned $1 \mathrm{RBs}$ (it is predefined in the code) at the first TTI. After all the flows have finished their data to be transmitted, five RBs have assigned for each flow as show in the figure4. 


\section{ISSN 2277-3061}

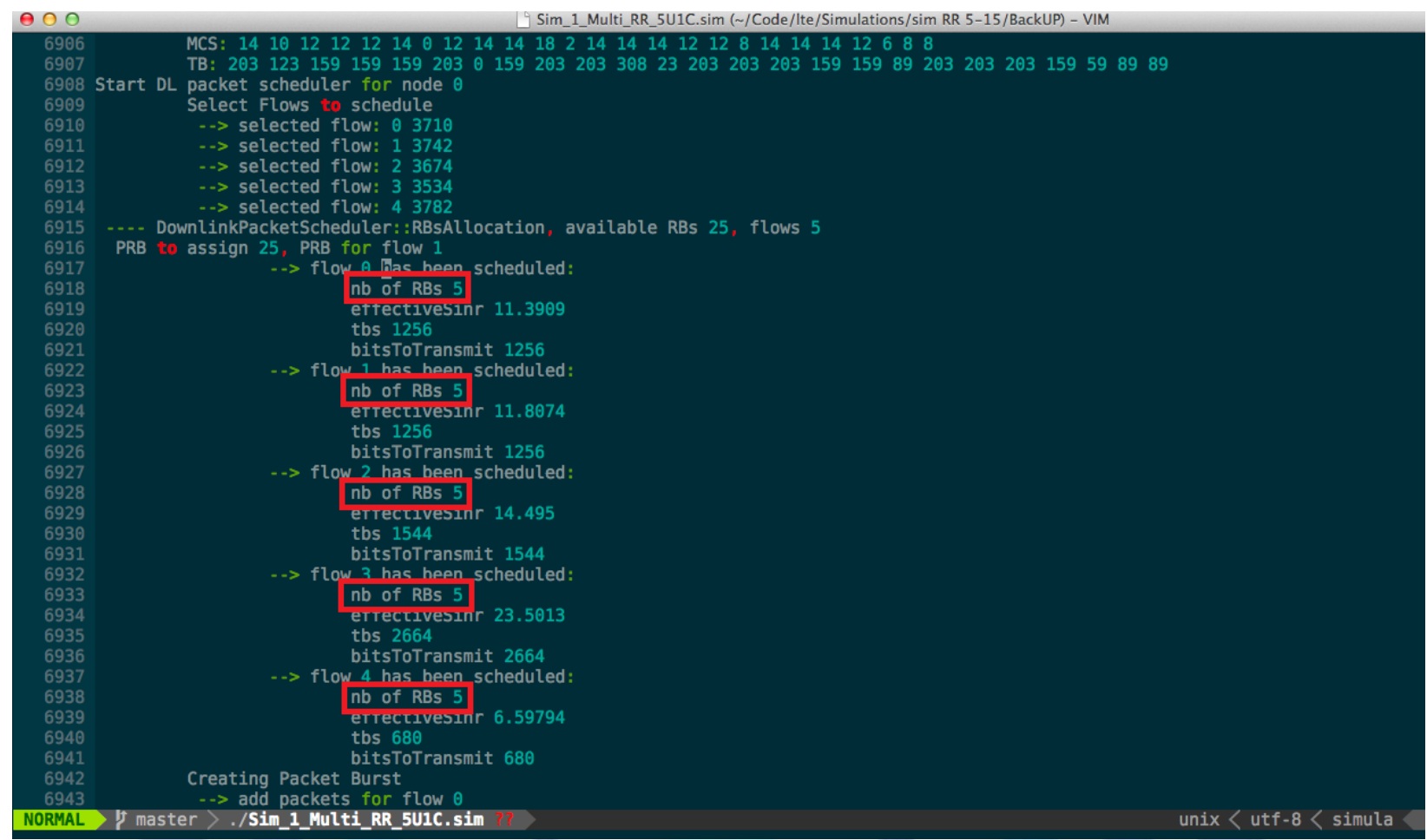

Fig3: Each flow allocate same numbers of RBs

\subsection{MaxRate algorithm}

MR depends on the metric value in order to allocate radio resources. The user with high metric will be scheduled while user with low metric will deprived from resources. Figure 4 shows the same scenario for RR has repeated to verify MR code is working.

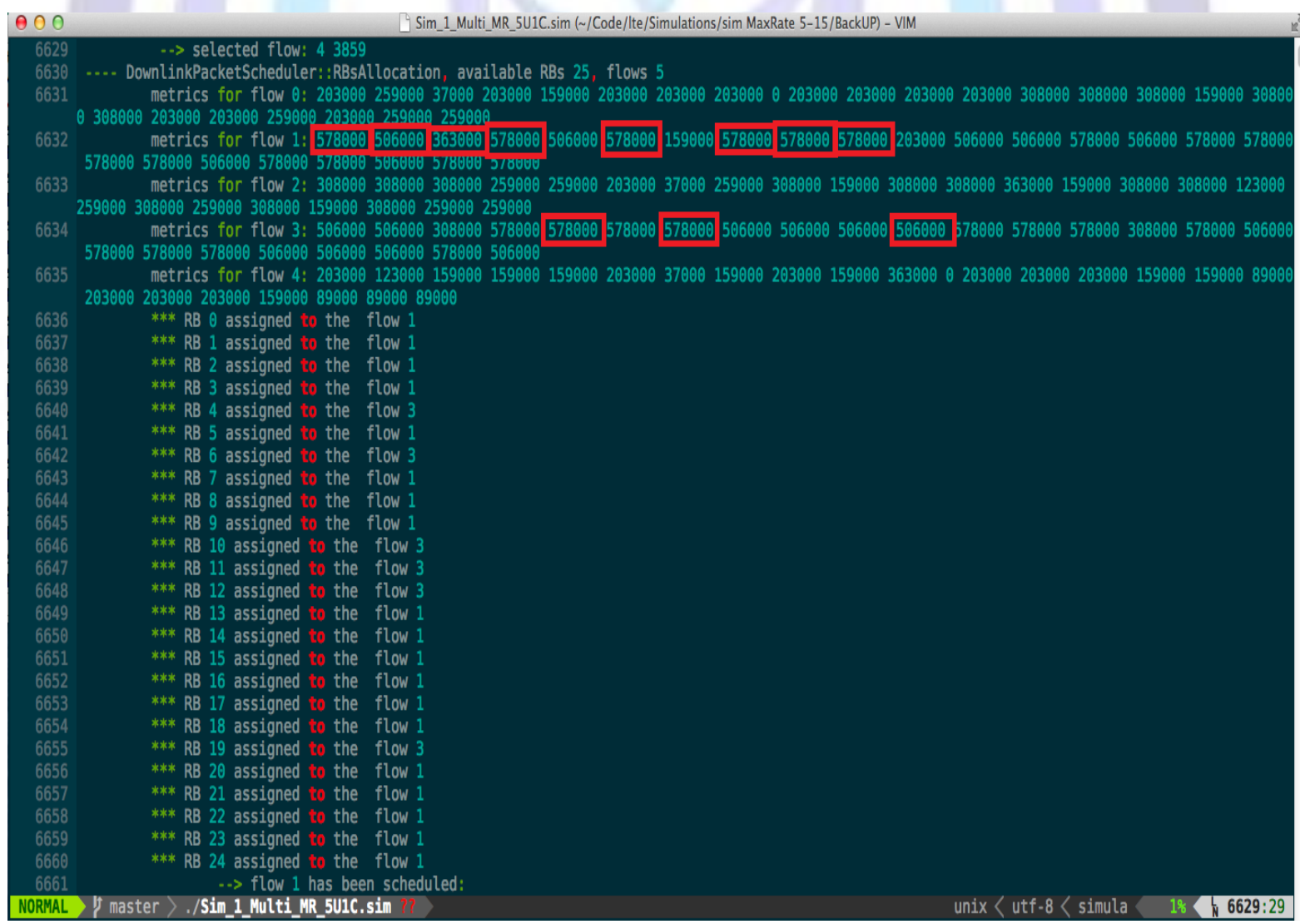

Fig4: MR code allocating RBs based on the highest metric 


\section{Simulation}

The following sections will show the simulations environment to evaluate the performance of thenew implemented algorithms with along with the current one in LTE-Sim.

\subsection{Simulation Environment for Packet scheduling algorithms}

A single eNodeB with fixed location is used. The bandwidth of $5 \mathrm{MHz}$ with 25 resources blocks is used. Other parameters are shown the Table 1.

Table 1. 3GPP LTE Downlink parameters

\begin{tabular}{|c|c|}
\hline Bandwidth & $5 \mathrm{MHz}$ \\
\hline Number of Sub-carriers & 300 \\
\hline Number of RBs & 25 \\
\hline Number of Sub-carriers per RB & $12 \mathrm{kHz}$ \\
\hline Sub-Carrier Spacing & $0.5 \mathrm{~ms}$ \\
\hline Slot Duration & $1 \mathrm{~ms}$ \\
\hline Scheduling Time (TTI) & 7 \\
\hline Slot & 120 \\
\hline
\end{tabular}

The number of users is set to 120 starting from $(80$ - 120). The traffic is selected to be RT (video streaming) and NRT (data traffic). Two simulations were tested for both speed $3 \mathrm{Km} / \mathrm{h}$ and $120 \mathrm{Km} / \mathrm{h}$.

\subsection{Simulations results and discussion}

\subsubsection{With $3 \mathrm{Km} / \mathrm{h}$ speed}

The evaluation of the five packet scheduling algorithms is tested with respect to the throughput, fairness, packet loss ratio, anddelay. Figure 5 and 6 show system throughput for both video and data traffic respectively. From both figures, it reveal the Max-Rate over perform both EXP/PF and M-LWDF. While RR and PF have low throughput in video and only RR is lower in data traffic as it does not depend on CQI report in allocating resources blocks.

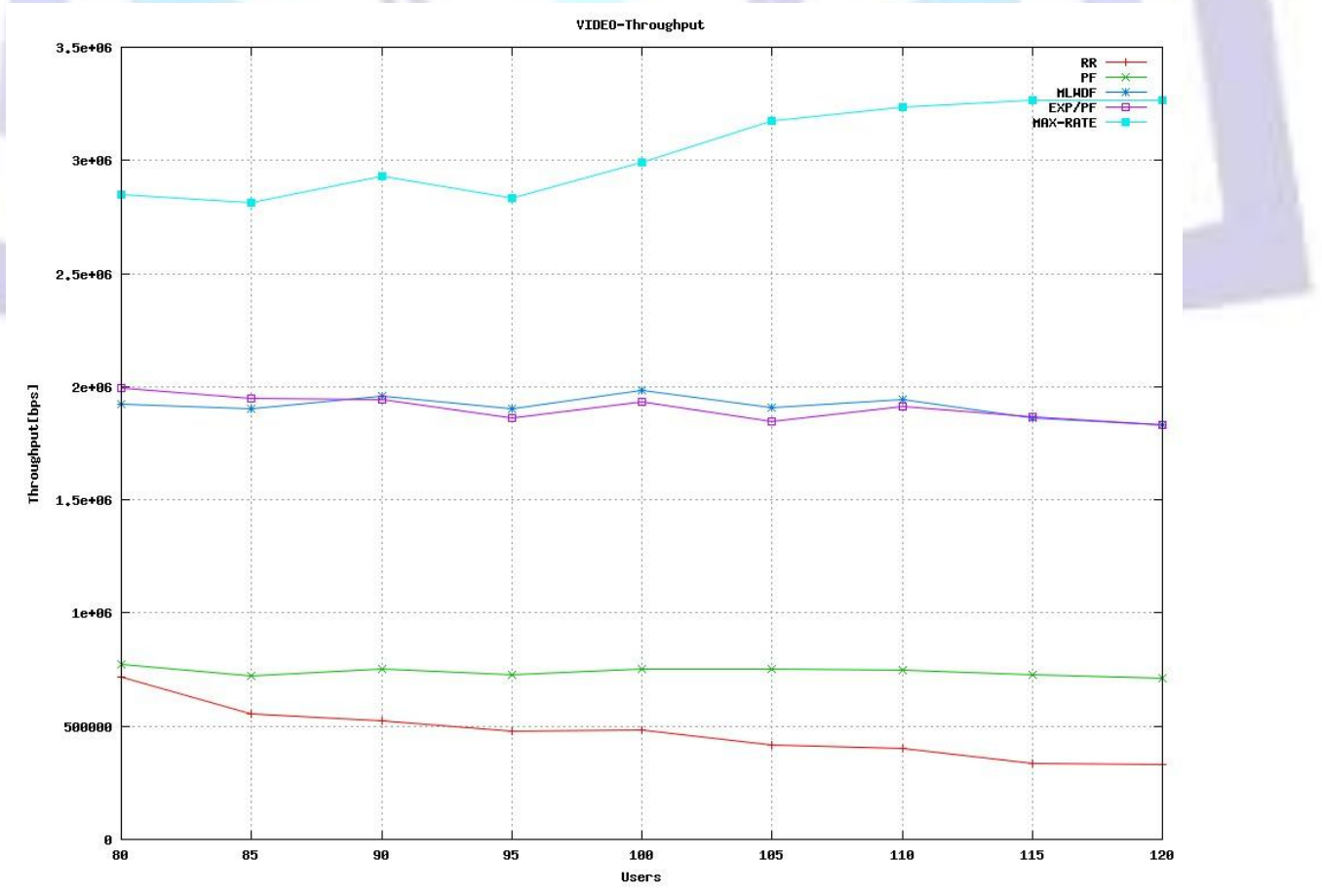

Fig5: Video System Throughput vs. Number of Users 


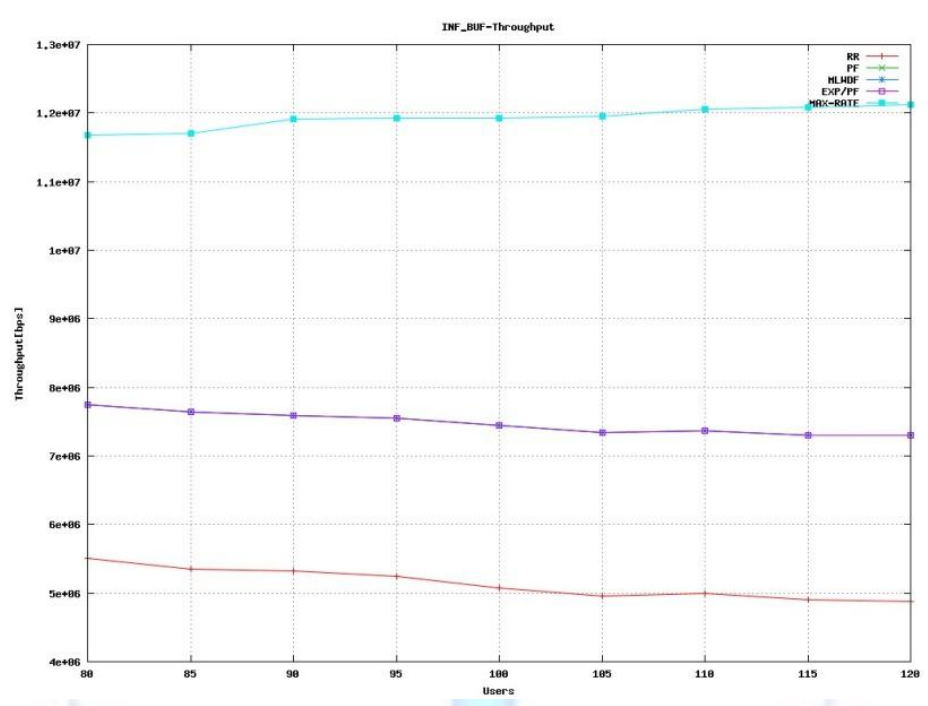

Fig6: Data traffic System Throughput vs. Number of Users

For packet loss ratio (PLR), figure 7 and 8 show that $R R$ has a significant high ratio while MR has low ratio. EXP/PF and MLWDF have almost the same value. The results of both speed almost the same with regarding to the high PLR for RR and low ratio for MR

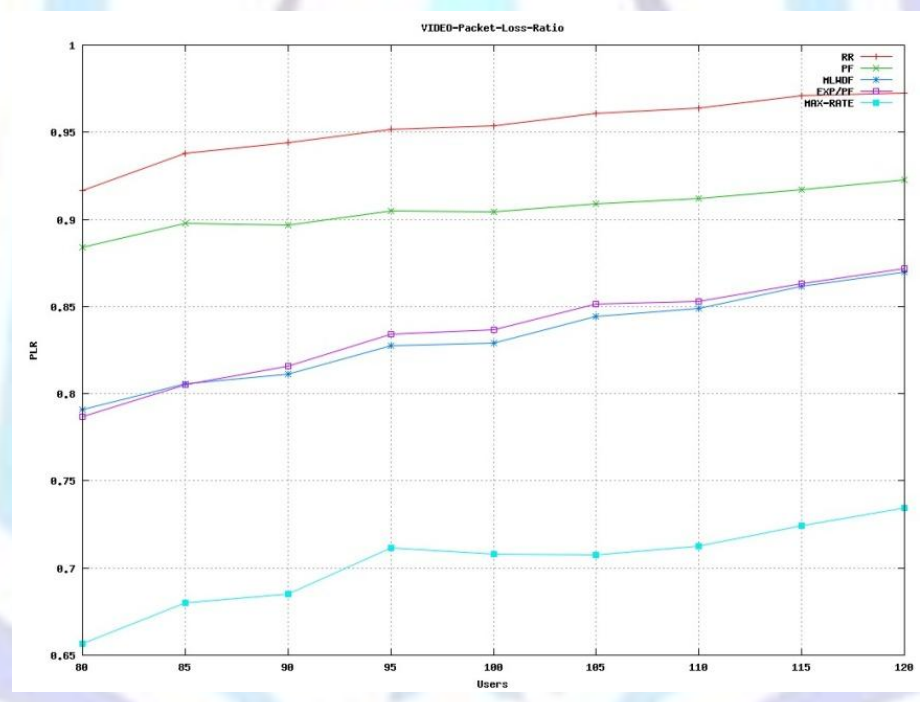

Fig7: Video Packet Loss Ratio vs. Number of Users

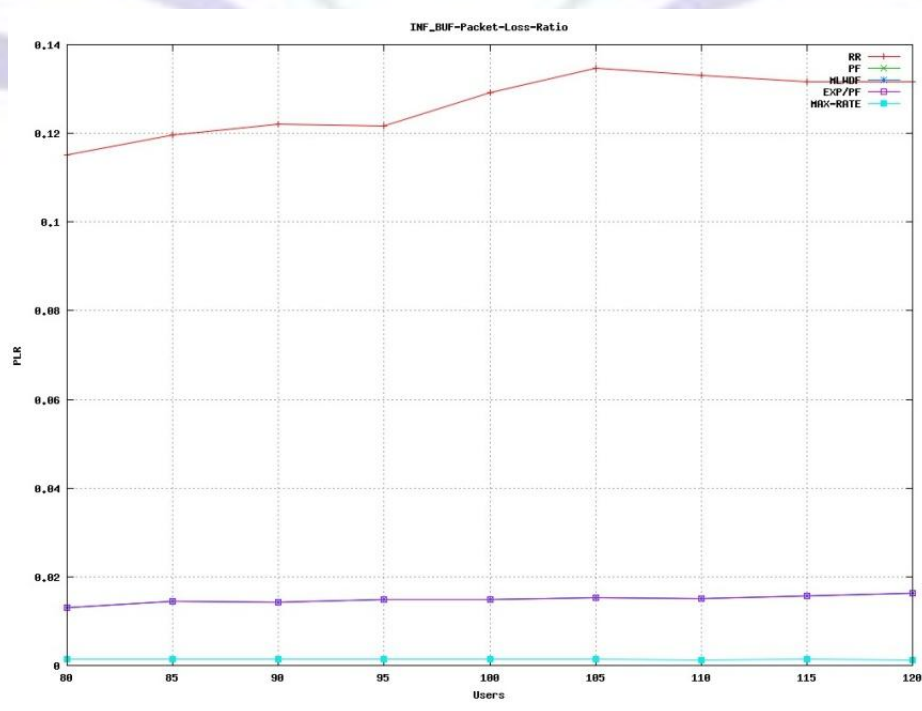

Fig8: Data trafficPacket Loss Ratio vs. Number of Users 
The fairness for all algorithms does not match with theoretical as RR must have the highest value. The results for both speeds show weird fairness results due to limitations in the simulation itself. Many efforts have been dedicated to verify and tuning it but unfortunately no change has been made.

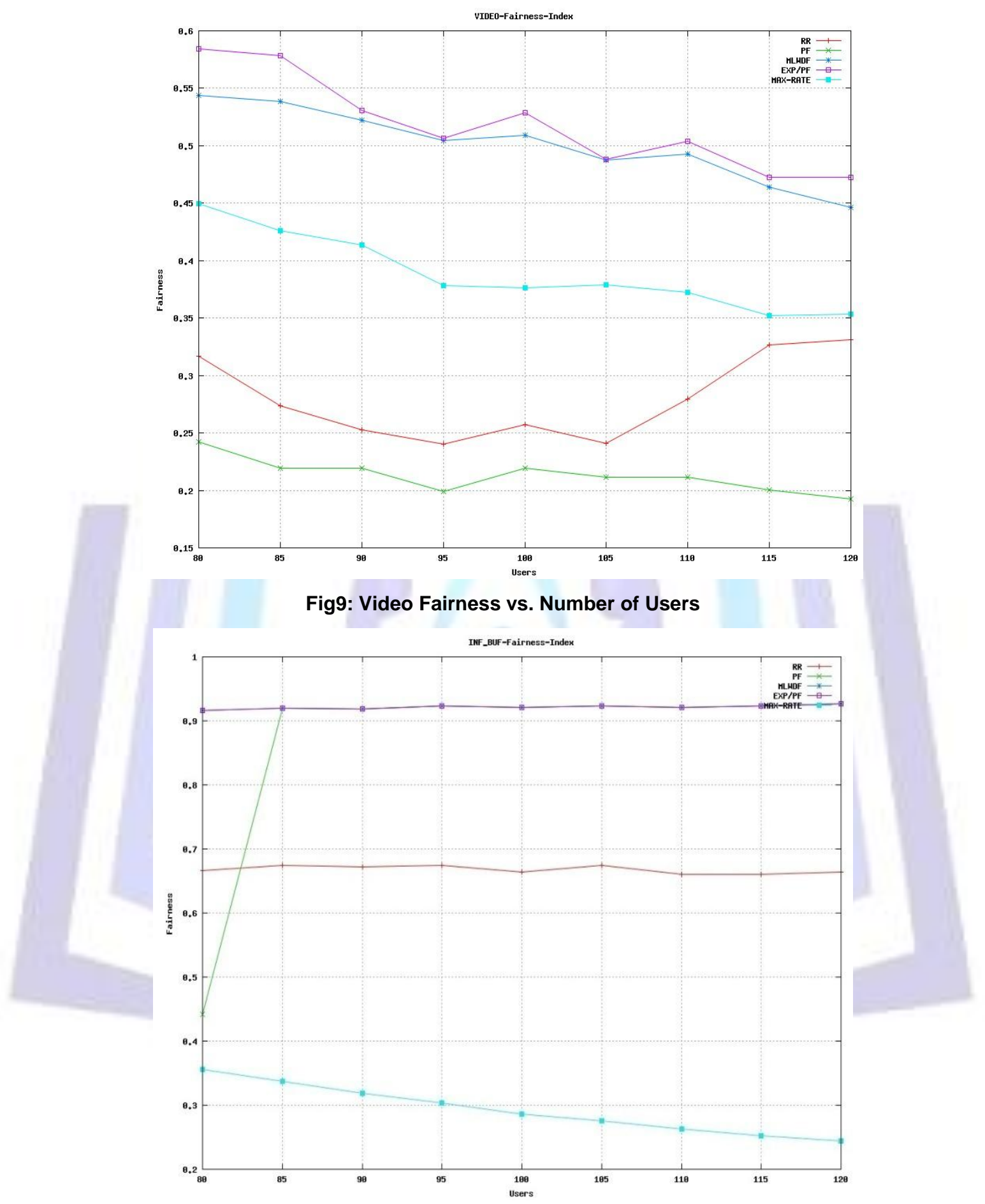

Fig10: Data traffic Fairness vs. Number of Users

\subsubsection{With $120 \mathrm{Km} / \mathrm{h}$ speed}

The through put with $120 \mathrm{Km} / \mathrm{h}$ speed has the same results with $3 \mathrm{Km} / \mathrm{h}$ speed for the video and slightly different in data traffic as shown in figure 11 and 12. 


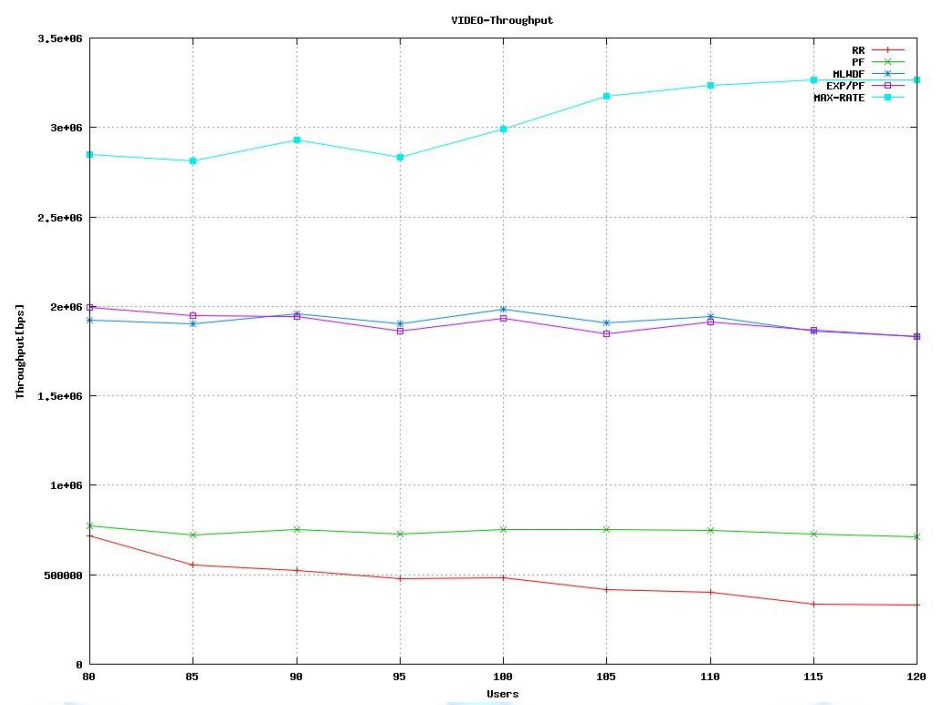

Fig11: Video System Throughput vs. Number of Users

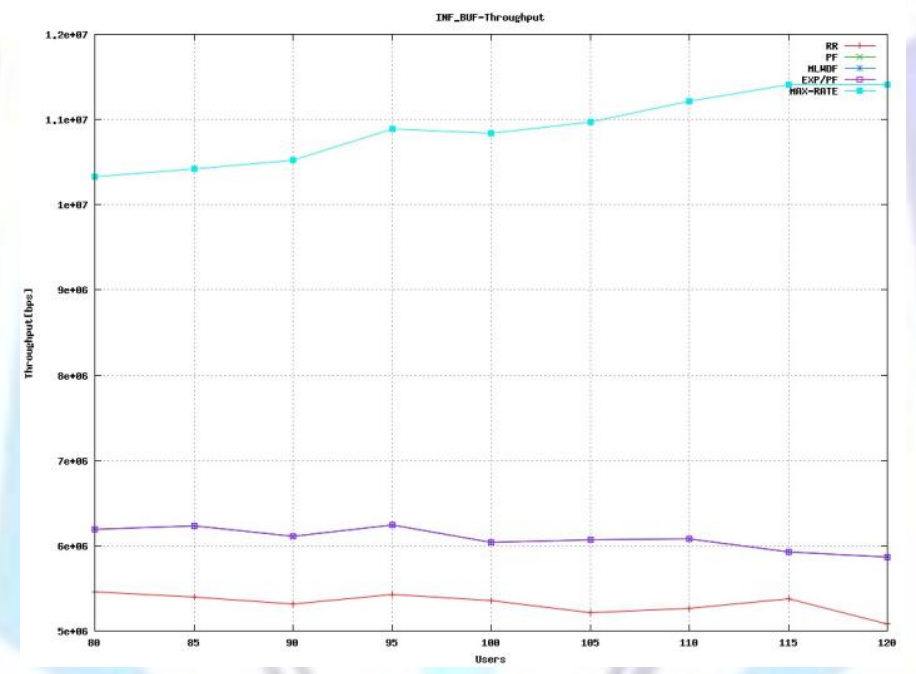

Fig12: Data traffic System Throughput vs. Number of Users

The PLR for both speed in video are slightly different but in data there is significant different. The RR has low PLR with compared with EXP/PF and MLWDF. This shows a weird result as RR has to have the highest value as it does not depend on the channel quality condition in allocating resources. The figures below show that.

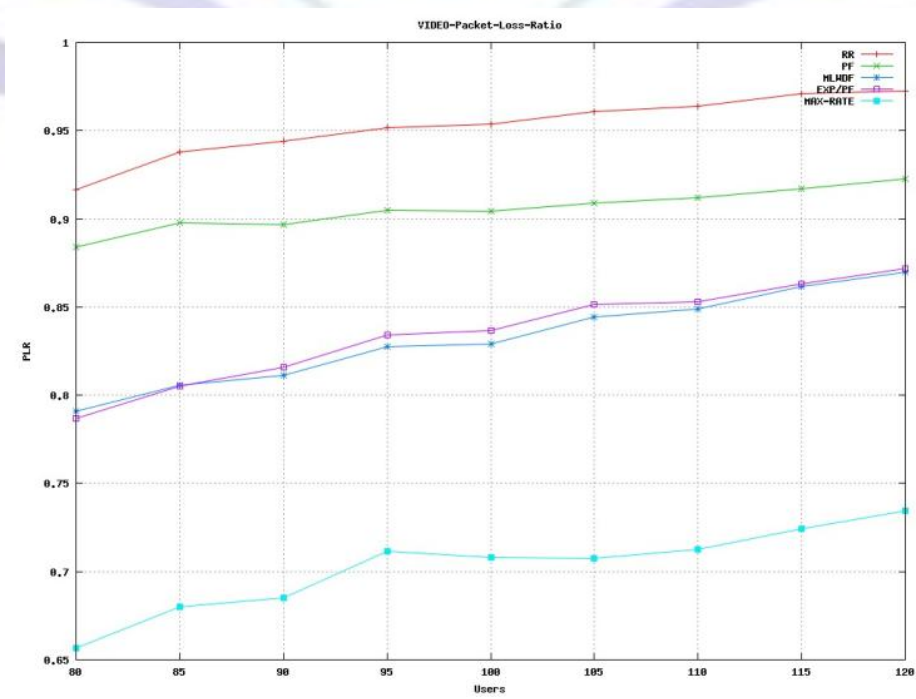

Fig13: Video Packet Loss Ratio vs. Number of Users 


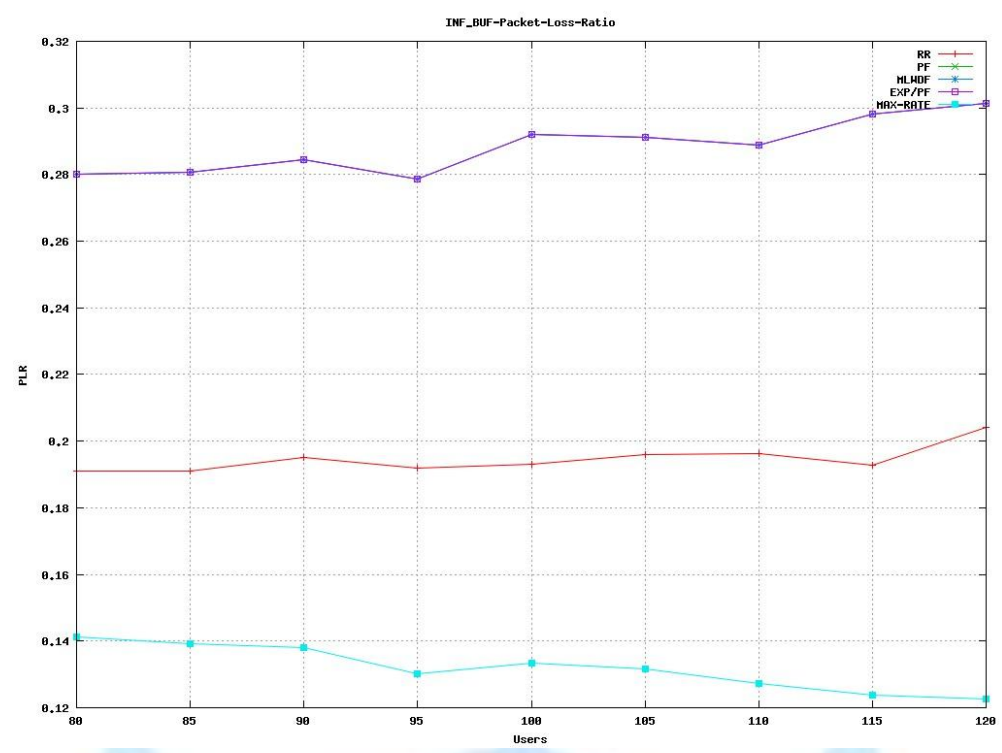

Fig14: Data traffic Packet Loss Ratio vs. Number of Users

The fairness result has the same issues for both speeds as shown in the figure 20 and 21:

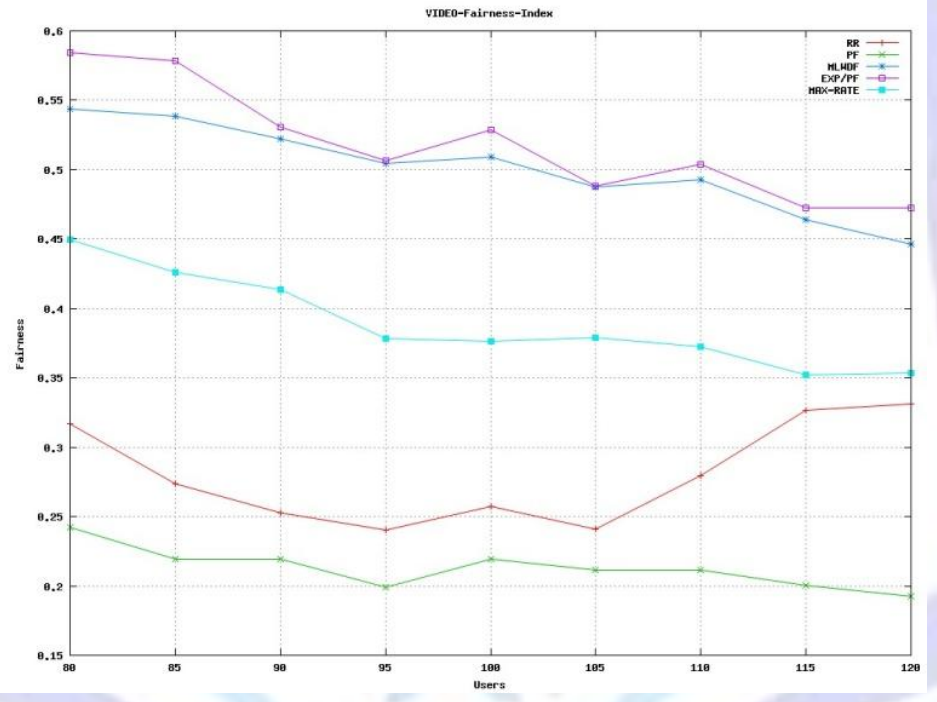

Fig15: Video Fairness vs. Number of Users

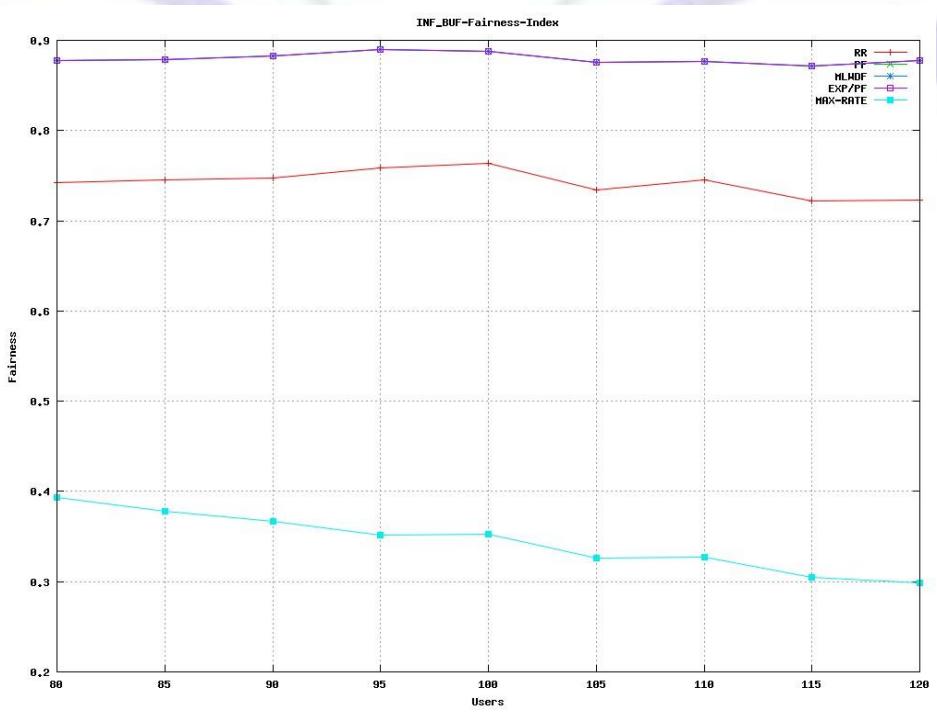

Fig16: Data traffic Fairness vs. Number of Users 


\section{Conclusion}

In this paper two packet scheduling algorithms are implemented to test the performance of real time and non real time services in LTE-Sim under 3GPP LTE system. It shows the strengths and weaknesses of these algorithms and some limitation in the LTE-Sim results regarding to fairness and PLR especially in scheduling algorithms. The MaxRate outperform other packet scheduling algorithms with respect to the throughput. The simulation program needs to be improved in order to get result closely to the theory.

\section{Reference}

[1] E. Dahlman, S. Parkvall, J. Skold, and P. Beming, 3G evolution: HSPA and LTE for mobile broadband: Academic press, 2010.

[2] C.-C. Lin, K. Sandrasegaran, H. Ramli, R. Basukala, R. Patachaianand, L. Chen, et al., "Optimization of handover algorithms in 3GPP long term evolution system," in Modeling, Simulation and Applied Optimization (ICMSAO), 2011 4th International Conference on, 2011, pp. 1-5.

[3] H. G. Myung, "Technical overview of 3GPP LTE," Polytechnic University of New York, 2008.

[4] F. Capozzi, G. Piro, L. A. Grieco, G. Boggia, and P. Camarda, "Downlink packet scheduling in Ite cellular networks: Key design issues and a survey," Communications Surveys \& Tutorials, IEEE, vol. 15, pp. 678-700, 2013.

[5] H. A. M. Ramli, R. Basukala, K. Sandrasegaran, and R. Patachaianand, "Performance of well known packet scheduling algorithms in the downlink 3GPP LTE system," in Communications (MICC), 2009 IEEE 9th Malaysia International Conference on, 2009, pp. 815-820.

[6] B. Tsybakov, "File transmission over wireless fast fading downlink," Information Theory, IEEE Transactions on, vol. 48, pp. 2323-2337, 2002.

[7] A. Jalali, R. Padovani, and R. Pankaj, "Data throughput of CDMA-HDR a high efficiency-high data rate personal communication wireless system," in Vehicular Technology Conference Proceedings, 2000. VTC 2000-Spring Tokyo. 2000 IEEE 51st, 2000, pp. 1854-1858 vol.3.

[8] M. Andrews, K. Kumaran, K. Ramanan, A. Stolyar, P. Whiting, and R. Vijayakumar, "Providing quality of service over a shared wireless link," Communications Magazine, IEEE, vol. 39, pp. 150-154, 2001.

[9] R. Jong-Hun, J. M. Holtzman, and K. Dong-Ku, "Scheduling of real/non-real time services: adaptive EXP/PF algorithm," in Vehicular Technology Conference, 2003. VTC 2003-Spring. The 57th IEEE Semiannual, 2003, pp. $462-466$ vol.1. 